\title{
Assessment of the Anthelmintic Efficacy of Albendazole in School Children in Seven Countries Where Soil- Transmitted Helminths Are Endemic
}

\author{
Jozef Vercruysse ${ }^{1 *}$, Jerzy M. Behnke ${ }^{2}$, Marco Albonico ${ }^{3}$, Shaali Makame Ame ${ }^{3}$, Cécile Angebault ${ }^{4}$, \\ Jeffrey M. Bethony ${ }^{5}$, Dirk Engels ${ }^{6}$, Bertrand Guillard ${ }^{4}$, Nguyen Thi Viet Hoa ${ }^{7}$, Gagandeep Kang ${ }^{8}$, Deepthi \\ Kattula ${ }^{8}$, Andrew C. Kotze ${ }^{9}$, James S. McCarthy ${ }^{10}$, Zeleke Mekonnen ${ }^{11}$, Antonio Montresor ${ }^{6}$, Maria \\ Victoria Periago ${ }^{5}$, Laurentine Sumo ${ }^{12}$, Louis-Albert Tchuem Tchuenté ${ }^{12}$, Dang Thi Cam Thach ${ }^{7}$, Ahmed \\ Zeynudin $^{11}$, Bruno Levecke'
}

1 Department of Virology, Parasitology and Immunology, Ghent University, Faculty of Veterinary Medicine, Merelbeke, Belgium, 2 School of Biology, University of Nottingham, Nottingham, United Kingdom, 3 Public Health Laboratory, Ivo de Carneri, Pemba Island, Zanzibar, Tanzania, 4 Institut Pasteur in Cambodia, Clinical Pathology Unit, Phnom Penh, Cambodia, 5 Instituto René Rachou, Fundação Oswaldo Cruz, Belo Horizonte, Minas Gerais, Brazil, 6 Department of Neglected Tropical Diseases, World Health Organization, Geneva, Switzerland, 7 National Institute for Malariology, Parasitology and Entomology, Hanoi, Vietnam, 8 Department of Gastrointestinal Sciences, Christian Medical College, Vellore, India, 9 Division of Livestock Industries, Commonwealth Scientific and Industrial Research Organisation, Brisbane, Australia, 10 Queensland Institute for Medical Research, University of Queensland, Herston, Australia, 11 Department of Medical Laboratory Sciences and Pathology, College of Public Health and Medical Sciences, Jimma University, Jimma, Ethiopia, 12 Centre for Schistosomiasis and Parasitology, Faculty of Sciences, University of Yaoundé I, Cameroon

\begin{abstract}
Background: The three major soil-transmitted helminths (STH) Ascaris lumbricoides, Trichuris trichiura and Necator americanus/Ancylostoma duodenale are among the most widespread parasites worldwide. Despite the global expansion of preventive anthelmintic treatment, standard operating procedures to monitor anthelmintic drug efficacy are lacking. The objective of this study, therefore, was to define the efficacy of a single 400 milligram dose of albendazole (ALB) against these three STH using a standardized protocol.

Methodology/Principal Findings: Seven trials were undertaken among school children in Brazil, Cameroon, Cambodia, Ethiopia, India, Tanzania and Vietnam. Efficacy was assessed by the Cure Rate (CR) and the Fecal Egg Count Reduction (FECR) using the McMaster egg counting technique to determine fecal egg counts (FEC). Overall, the highest CRs were observed for A. lumbricoides (98.2\%) followed by hookworms (87.8\%) and T. trichiura (46.6\%). There was considerable variation in the CR for the three parasites across trials (country), by age or the pre-intervention FEC (pre-treatment). The latter is probably the most important as it had a considerable effect on the CR of all three STH. Therapeutic efficacies, as reflected by the FECRs, were very high for A. lumbricoides (99.5\%) and hookworms (94.8\%) but significantly lower for $T$. trichiura (50.8\%), and were affected to different extents among the 3 species by the pre-intervention FEC counts and trial (country), but not by sex or age.

Conclusions/Significance: Our findings suggest that a FECR (based on arithmetic means) of $>95 \%$ for $A$. lumbricoides and $>90 \%$ for hookworms should be the expected minimum in all future surveys, and that therapeutic efficacy below this level following a single dose of ALB should be viewed with concern in light of potential drug resistance. A standard threshold for efficacy against $T$. trichiura has yet to be established, as a single-dose of ALB is unlikely to be satisfactory for this parasite.
\end{abstract}

Trial Registration: ClinicalTrials.gov NCT01087099

Citation: Vercruysse J, Behnke JM, Albonico M, Ame SM, Angebault C, et al. (2011) Assessment of the Anthelmintic Efficacy of Albendazole in School Children in Seven Countries Where Soil-Transmitted Helminths Are Endemic. PLoS Negl Trop Dis 5(3): e948. doi:10.1371/journal.pntd.0000948

Editor: Simon Brooker, London School of Hygiene \& Tropical Medicine, United Kingdom

Received May 18, 2010; Accepted December 15, 2010; Published March 29, 2011

Copyright: (c) 2011 Vercruysse et al. This is an open-access article distributed under the terms of the Creative Commons Attribution License, which permits unrestricted use, distribution, and reproduction in any medium, provided the original author and source are credited.

Funding: The study was funded in part by the WHO (www.who.int; Ref Nr 2008/12964/0; Reg File Nr P2-APW002). BL is funded by the Fund for Scientific Research-Flanders (Belgium) (F.W.O.-Vlaanderen) (www.fwo.be; Ref Nr G.0853.09). The funders had no role in study design, data collection and analysis, decision to publish, or preparation of the manuscript.

Competing Interests: The authors have declared that no competing interests exist.

*E-mail: Jozef.Vercruysse@ugent.be

\section{Introduction}

The three major Soil-Transmitted Helminths (STH), Ascaris lumbricoides (roundworm), Trichuris trichiura (whipworm) and Necator americanus/Ancylostoma duodenale (the hookworms) are amongst the most widespread parasites worldwide. An estimated 4.5 billion individuals are at risk of STH infection and more than one billion individuals are thought to be infected, of whom 450 million suffer morbidity from their infection, the majority of who are children. An additional 44 million infected pregnant women suffer 


\section{Author Summary}

Soil-transmitted helminths (roundworms, whipworms and hookworms) infect millions of children in (sub)tropical countries, resulting in malnutrition, growth stunting, intellectual retardation and cognitive deficits. Currently, there is a need to closely monitor anthelmintic drug efficacy and to develop standard operating procedures, as highlighted in a World Health Organization-World Bank meeting on "Monitoring of Drug Efficacy in Large Scale Treatment Programs for Human Helminthiasis" in Washington DC at the end of 2007. Therefore, we have evaluated the efficacy of a commonly used treatment against these parasitic infections in school children in Africa, Asia and South-America using a standardized protocol. In addition, different statistical approaches to analyzing the data were evaluated in order to develop standardized procedures for data analysis. The results demonstrate that the applied treatment was highly efficacious against round- and hookworms, but not against whipworms. However, there was large variation in efficacy across the different trials which warrants further attention. This study also provides new insights into the statistical analysis of efficacy data, which should be considered in future monitoring and evaluation studies of large scale anthelmintic treatment programs. Finally, our findings emphasize the need to update the World Health Organization recommended efficacy threshold for the treatment of STH.

significant morbidity and mortality due to hookworm-associated anemia. Approximately 135,000 deaths occur per year, mainly due to infections with hookworms or A. lumbricoides [1].

The most widely implemented method of controlling STH infections is through periodic administration of anthelmintics. Rather than aiming to achieve eradication, current control programs are focused on reducing infection intensity and transmission potential, primarily to reduce morbidity and avoid mortality associated with the disease [2]. The benzimidazole (BZ) drugs, i.e. albendazole (ALB) and mebendazole, are the most widely used drugs for the control of STH. While both show broadspectrum anthelmintic activity, for hookworms a single dose of ALB is more effective than mebendazole [3].

The scale up of chemotherapy programs that is underway in various parts of Africa, Asia and South America, particularly targeting school children, is likely to exert increasing drug pressure on parasite populations, a circumstance that is likely to favor parasite genotypes that can resist anthelmintic drugs. Given the paucity of suitable alternative anthelmintics it is imperative that monitoring programs are introduced, both to assess progress and to detect any changes in therapeutic efficacy that may arise from the selection of worms carrying genes responsible for drug resistance. The well documented occurrence of resistance to anthelmintics in nematode populations of livestock [4], highlights the potential for frequent treatments used in chemotherapy programs to select drug resistant worms. Such an eventuality threatens the success of treatment programs in humans, both at individual and community levels [5]. Although some small scale studies [6,7], have suggested emerging drug resistance in human $\mathrm{STH}$, these studies should be interpreted with some caution, since suboptimal efficacy could have been due to factors other than drug resistance. Moreover, although for the BZ drugs there are many published studies reporting the Cure Rate (CR) and the Fecal Egg Count Reduction (FECR), the two most widely used indicators for assessing the efficacy of an anthelmintic in human medicine, comparison of such studies is difficult, largely because there is no widely accepted standard operating procedure for undertaking such trials [8]. Published studies are confounded by methodological variations including treatment regimens, poor quality of drugs, differing statistical analyses used to calculate therapeutic efficacy, as well as a range of other problems in study design, such as small sample size, diagnostic methods, variation in pre-intervention infection intensities and confounding factors related to geographical locations. Such variation among studies greatly complicates direct comparison [3]. A World Health OrganizationWorld Bank (WHO-WB) meeting on "Monitoring of Drug Efficacy in Large Scale Treatment Programs for Human Helminthiasis", held in Washington DC at the end of 2007, highlighted the need to closely monitor anthelmintic drug efficacy and to develop standard operating procedures for this purpose. In a systematic meta-analysis of published single-dose studies, Keiser and Utzinger [8], confirmed that there was a paucity of high quality trials, and that the majority of trials were carried out more than 20 years ago. They recommended that well-designed, adequately powered, and rigorously implemented trials should be undertaken to provide current data regarding the efficacy of anthelmintics against the main species of STH. These should be designed to establish benchmarks (including standard operating procedures) for subsequent monitoring of drug resistance.

The objective of the present work was to validate a standard protocol that has been developed for monitoring efficacy of anthelmintics against STH. To give the study wide relevance, we conducted the trial in seven populations in different geographic locations in Brazil, Cameroon, Cambodia, Ethiopia, India, Tanzania and Vietnam. In each of the study sites, different epidemiologic patterns of infection prevail, including different combinations of STH. We assessed the efficacy of a single dose $(400 \mathrm{mg})$ of ALB in terms of the CR and the FECR in school children between 14 and 30 days following treatment. The McMaster egg counting technique was used in a standardized fashion, with rigorous quality control. Levecke et al. [9] reported that the McMaster holds promise as a standardized method on account of its applicability for quantitative screening of large numbers of subjects. This method is the recommended method for measuring fecal egg counts (FEG) when performing FECR for the detection of anthelmintic resistance in veterinary medicine $[10,11]$.

\section{Methods}

\section{Study sites}

This study was carried out in seven different countries covering Africa (Cameroon, Ethiopia and Tanzania), Asia (Cambodia, India and Vietnam) and South-America (Brazil). However, it is important to note, that while we refer to individual countries to identify results from particular trials, we do not make any conclusions about any country as such. Here, names of countries are used only to distinguish between 7 separate trials that were conducted in 7 geographically disparate regions of the world. In total ten study sites with varying STH and treatment history were included. These seven STH endemic countries were selected because of the presence of investigator groups with previous extensive experience in the diagnosis and control of STH. Table 1 provides their specific locations (district/province/state) and treatment history. Both species of hookworms $(\mathcal{N}$. americanus and A. duodenale) were present in all study sites in varying degree with the exception of Brazil where only $\mathcal{N}$. americanus was present.

\section{Trial design}

During the pre-intervention survey, school children aged 4 to 18 years at the different study sites were asked to provide a stool sample. For the initial sampling the aim was to enroll at least 250 
Table 1. The location and treatment history of the ten study sites.

\begin{tabular}{lll}
\hline Country & & \\
\hline Brazil & District/Province/State & Treatment history \\
Cambodia & Minas Gerais & LSAT since 2007 (ALB) \\
Cameroon & Kratie & LSAT since 1997, last in 2007 (MBD) \\
& Loum & LSAT (MBD/ALB) since 1999, last in 2008 (MBD) \\
Ethiopia & Yoyo & No LSAT \\
India & Jimma & No LSAT \\
& Vellore & LSAT, since 2001, last in 2008 (ALB) \\
Tanzania (Zanzibar) & Thiruvanamalai & No LSAT \\
Vietnam & Pemba Island & LSAT since 1994, last in 2006 (PZQ, IVM, ALB) \\
& Thái Nguyên & LSAT since 2005 \\
\hline
\end{tabular}

LSAT: large scale anthelmintic treatment, MBD: mebendazole, PZQ: praziquantel, IVM: ivermectine, ALB: albendazole.

doi:10.1371/journal.pntd.0000948.t001

infected children with a minimum of 150 eggs per gram of feces (EPG) for at least one of the STH. This sample size was selected based on statistical analysis of study power, using random simulations of correlated over-dispersed FEG data reflecting the variance-covariance structure in a selection of real FEC data sets. This analysis suggested that a sample size of up to 200 individuals $(\alpha=0.05$, power $=80 \%)$ was required to detect a 10 percentage point drop from a null efficacy of $\sim 80 \%$ (mean percentage FEC $\Delta$ per individual) over a wide range of infection scenarios. Standard power analyses for proportions also indicated that the detection of a $\sim 10$ percentage point drop from a null cure rate required sample sizes up to 200 (the largest samples being required to detect departures from null efficacies of around $50 \%$ ). Given an anticipated non-compliance rate of $25 \%$, a sample of 250 individuals with $>150$ EPG pre-treatment was therefore considered necessary at each study site.

Fecal samples were processed using the McMaster technique (analytic sensitivity of 50 EPG) for the detection and the enumeration of infections with $A$. lumbricoides, $T$. trichiura and hookworms [9]. None of the samples were preserved. Samples which could not be processed within 24 hours were kept at $4^{\circ} \mathrm{C}$. A single dose of $400 \mathrm{mg}$ ALB (Zentel) from the same manufacturer (GlaxoSmithKline Pharmaceuticals Limited, India) and same lot (batch number: B.N ${ }^{\circ}$ : L298) was used at all trial sites. No placebo control subjects were included in the trial for ethical and operational reasons. Between 14 to 30 days after the preintervention survey, stool samples were collected from the treated subjects and processed by the McMaster technique. All of the trials were carried out in a single calendar year (2009). Subjects who were unable to provide a stool sample at follow-up, or who were experiencing a severe concurrent medical condition or had diarrhea at time of the first sampling, were excluded from the study. The participation, the occurrence of STH and sample submission compliance for pre- and post-intervention surveys are summarized in Figure 1.

\section{The McMaster counting technique}

The McMaster counting technique (McMaster) was based on the modified McMaster described by the Ministry of Agriculture, Fisheries and Food (UK; 1986) [12]. Two grams of fresh stool samples were suspended in $30 \mathrm{ml}$ of saturated salt solution $($ density $=1.2)$. The suspension was poured three times through a wire mesh to remove large debris. Then $0.15 \mathrm{ml}$ aliquots were added to each of the 2 chambers of a McMaster slide. Both chambers were examined under a light microscope using a 100x magnification and the FEC for each helminth species was obtained by multiplying the total number of eggs by 50 .

\section{Statistical analysis}

The efficacy of the treatment for each of the three STH was evaluated qualitatively based on the reduction in infected children (CR) and quantitatively based on the reduction in fecal egg counts (FECR). The outcome of the FECR was calculated using three formulae. The first two formulae were based on the mean (arithmetic/geometric) of the pre- and post-intervention fecal egg count (FEG) ignoring the individual variability, whereas the third formula represented the mean of the reduction in the FEC per subject. The latter is the only quantitative indicator of efficacy for which the importance of confounding factors can be assessed by statistical analysis.

$\operatorname{FECR}(1)=100 \% \times$

arithmetic mean(pre-intervention FEC) - arithmetic mean(post-intervention FEC) arithmetic mean(pre-intervention FEC)

$\operatorname{FECR}(2)=100 \% \times$

geometric mean(pre-intervention FEC) - geometric mean(post-intervention FEC) geometric mean(pre-intervention FEC)

$$
\begin{aligned}
& \operatorname{FECR}(3)=100 \% \times \\
& \text { arithmetic mean }\left(\frac{[\text { pre-interevention FEC }]-[\text { post-intervention FEC }]}{\text { pre-interevention FEC }}\right)
\end{aligned}
$$

The GR and the FECR (1-3) outputs were calculated for the different trials, both sexes, age classes (A: 4-8 years; B: 9-13 years and C: 14-18 years) and for the level of egg excretion intensity at the pre-intervention survey. These levels corresponded to the low, moderate and high intensities of infection as described Montresor et al. [13] For A. lumbricoides these were 1-4,999 EPG, 5,00049,999 EPG and >49,999 EPG; for T. trichiura these levels were 1999 EPG, 1000-9,999 EPG and >9,999 EPG; and for hookworms these were 1-1,999 EPG, 2,000-3,999 EPG and >3,999 EPG, respectively.

In addition, the robustness of the three FECR formulae was explored by comparing the FEC reduction rate obtained from all 


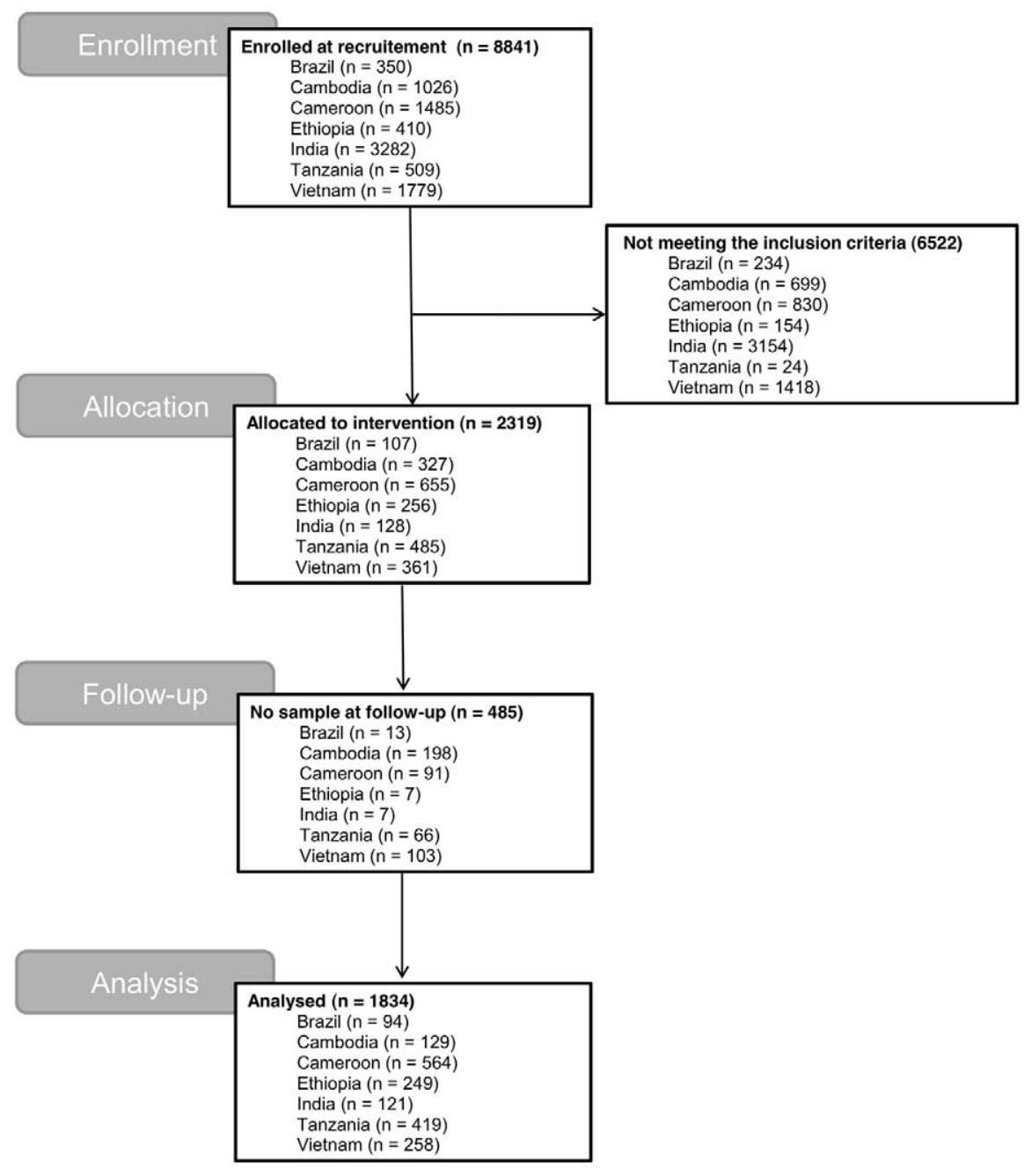

Figure 1. The participation, occurrence of STH and sample submission compliance for pre- and post-intervention surveys. Subjects who were not able to provide a sample for the follow-up, or who were experiencing a severe current medical condition or had diarrhea at the time of the first sampling were excluded from the trial. doi:10.1371/journal.pntd.0000948.g001

samples containing STH and those obtained from samples containing more than 150 EPG as recommended in the anthelmintic resistance guidelines of the World Association for the Advancement of Veterinary Parasitology [9]. Finally, putative factors affecting the GR and the FECR (3) were evaluated. For the CR, generalized linear models (binomial error) were built with the test result (infected /uninfected) as the outcome, 'trial' (7 levels: trials in Brazil, Cambodia, Cameroon, Ethiopia, India, Tanzania and Vietnam) and 'sex' (2 levels: female and male) as factors, and 'age' and the log transformed pre-intervention FEC as covariates. Full factorial models were evaluated by the backward selection procedure using the likelihood ratio test of $\chi^{2}$. Finally, the CR for each of the observed values of the covariate and factor was calculated based on these models (The R Foundation for Statistical Computing, version 2.10.0 [14]). For analysis of the data from FECR (3), non-parametric methods were used, because models based on parametric statistics, even with negative binomial error structures, or based on transformed data would not converge satisfactorily as a consequence of the high proportion of FEC with zero EPG. Hence, the impact of the factors 'trial' and 'sex' were assessed by the Kruskal-Wallis test (for more than 2 group comparisons) and the Mann-Whitney $\mathrm{U}$ test, respectively. The correlation between the outputs of FECR (3) and the covariates (age and pre-intervention FEC) was estimated by the Spearman rank order correlation coefficient (SAS 9.1.3, SAS Institute Inc.; Cary, NG, USA).

\section{Ethics statement}

The overall protocol of the study was approved by the Ethics committee of the Faculty of Medicine, Ghent University (Nr B67020084254) and was followed by a separate local ethical approval for each study site. For Brazil, approval was obtained from the Institutional Review Board from Centro de Pesquisas René Rachou (Nr 21/2008), for Cambodia from the National Ethic Commitee for Health Research, for Cameroon from the National Ethics Committee (Nr 072/CNE/DNM08), for Ethiopia from the Ethical Review Board of Jimma University, for India from the Institutional Review Board of the Christian Medical College (Nr 6541), for Tanzania (Nr 20) from the Zanzibar Health Research Council and the Ministry of Health and Social Welfare, for Vietnam by the Ministry of Health of Vietnam. An informed consent form was signed by the parents of all subjects included in the study. This clinical trial was registered under the ClinicalTrials.gov Identifier NCT01087099. 


\section{Results}

\section{The cure rate $(C R)$}

Overall, the highest CRs were observed for A. lumbricoides $(98.2 \%)$, followed by hookworm $(87.8 \%)$ and T. trichiura $(46.6 \%)$. However, as shown in Table 2, the CRs varied across the different trials, age classes and pre-intervention FEC levels. The differences in CRs between trials were most pronounced for $\mathcal{T}$. trichiura, ranging from 21.0 (Tanzania) to $88.9 \%$ (India). The T. trichiura CRs of $100 \%$ for the trials in Brazil and Cambodia are not considered here as they were based on only 1 and 2 individuals, respectively. For hookworms and $A$. lumbricoides, the CRs varied from 74.7 (India) to $100 \%$ (Vietnam) and from 96.4 (Tanzania) to 99.3\% (Ethiopia and Cameroon), respectively. The CRs for $A$. lumbricoides in Cambodia (100\%) and India (95.2\%) are not considered here as they were based on fewer than 50 individuals. The CRs increased over the three age classes (A. lumbricoides: 95.8 to $100 \%$; T. trichiura: 44.7 to $54.1 \%$ ), except for hookworms where the CRs ranged from 86.1 to 88.3 , and then to $87.5 \%$. For each of the three STH, there was a decline in the GR with increasing levels of infection intensities at the pre-intervention survey. The largest drop was observed for T. trichiura, which decreased from 53.9 to $12.5 \%$. For the two other STH, the drop in the CR was less pronounced, ranging from 88.6 to $76.9 \%$ for hookworms and only from 98.3 to $95 \%$ for $A$. lumbricoides. The observed differences between sexes were negligible for all three STH.

Differences in GR by trial, age and pre-intervention FEG are illustrated in Figure 2. The variability in the CR of the three

Table 2. The cure rate $(C R)$ for treatment with a single dose of albendazole against soil-transmitted helminths.

\begin{tabular}{|c|c|c|c|c|c|c|}
\hline & \multicolumn{2}{|c|}{$\begin{array}{l}\text { A. } \\
\text { lumbricoides }\end{array}$} & \multicolumn{2}{|c|}{ T. trichiura } & \multicolumn{2}{|c|}{ Hookworms } \\
\hline & $\mathbf{n}$ & CR (\%) & $\mathbf{N}$ & CR (\%) & $\mathbf{n}$ & CR (\%) \\
\hline \multicolumn{7}{|l|}{ Country } \\
\hline Brazil & 50 & 98.0 & $1^{*}$ & 100 & 52 & 88.5 \\
\hline Cambodia & $5^{*}$ & 100 & $2^{*}$ & 100 & 127 & 87.4 \\
\hline Cameroon & 298 & 99.3 & 386 & 47.4 & 140 & 87.1 \\
\hline Ethiopia & 151 & 99.3 & 105 & 85.7 & 91 & 98.9 \\
\hline India & $21^{*}$ & 95.2 & $18^{*}$ & 88.9 & 95 & 74.7 \\
\hline Tanzania & 279 & 96.4 & 396 & 21.0 & 349 & 86.8 \\
\hline Vietnam & 148 & 98.6 & 138 & 81.2 & 58 & 100 \\
\hline \multicolumn{7}{|l|}{ Age class } \\
\hline A (4-8) & 215 & 95.8 & 219 & 44.7 & 173 & 86.1 \\
\hline B (9-13) & 669 & 98.8 & 753 & 46.3 & 643 & 88.3 \\
\hline$C(14-18)$ & 68 & 100 & 74 & 54.1 & 96 & 87.5 \\
\hline \multicolumn{7}{|l|}{ Sex } \\
\hline Female & 462 & 98.1 & 503 & 48.5 & 393 & 89.1 \\
\hline Male & 490 & 98.4 & 543 & 44.8 & 519 & 86.9 \\
\hline \multicolumn{7}{|c|}{ Pre-intervention infection intensity } \\
\hline Low & 662 & 98.3 & 823 & 53.9 & 859 & 88.6 \\
\hline Moderate & 270 & 98.1 & 215 & 19.5 & 40 & 75.0 \\
\hline High & 20 & 95.0 & 8 & 12.5 & 13 & 76.9 \\
\hline Total & 952 & 98.2 & 1046 & 46.6 & 912 & 87.8 \\
\hline
\end{tabular}

parasites was significantly associated with these three factors (predictive value $>75 \%$ ). The pre-intervention FEG was probably the most important as it had a considerable effect on the $\mathrm{CR}$ of $A$. lumbricoides $\left(\chi^{2}{ }_{1}=4.14, p<0.05\right), \quad T$. trichiura $\left(\chi^{2}{ }_{1}=66.3, p<0.0001\right)$ and hookworms $\left(\chi_{1}^{2}=11.9, p<0.001\right)$. Age only contributed to variation in the $\mathrm{CR}$ of $A$. lumbricoides $\left(\chi^{2}{ }_{1}=6.8, p<0.01\right)$. Differences among the trials (countries) in the CR were observed for T. trichiura $\left(\chi^{2}{ }_{3}=33.8, p<0.0001\right)$ and hookworms $\left(\chi^{2}{ }_{6}=35.1, p<0.0001\right)$, but not for $A$. lumbricoides. In addition, there was an interaction between the pre-intervention FEC for $A$. lumbricoides $\left(\chi^{2}{ }_{1}=4.7, p<0.05\right)$ and for $T$. trichiura $\left(\chi^{2}{ }_{3}=18.4, p<0.0005\right)$ with age and trial (country) respectively (lines cross one another). The impact of pre-intervention FEC on the $\mathrm{CR}$ of $A$. lumbricoides was more pronounced for older individuals than younger ones. For $T$. trichura the effect of preintervention FEC varied considerably across the trials conducted in the different countries, particularly for the trial in Ethiopia where the CR dropped from almost 100 to nearly $0 \%$ as the preintervention FEC increased.

\section{Comparison of different formulae for assessing FECR}

The pre-intervention FEC for the different STH ranged from 50 to 170,500 EPG for $A$. lumbricoides (arithmetic mean $=6877$ EPG), from 50 to 23,200 EPG for T. trichiura (arithmetic mean $=824 \mathrm{EPG}$ ) and from 50 to $13,800 \mathrm{EPG}$ for hookworm (arithmetic mean $=650 \mathrm{EPG}$ ). The data in Table 3 show that there was considerable variation in the arithmetic means of the FEC from the trial groups in the 7 participating countries for each of the three STH species. As illustrated in Figure 3, pre-intervention FEC were highly aggregated among the subjects, and high FEC were only observed in relatively few subjects.

The FEC reduction rate calculated using all three formulae (based on FECR 1-3) in turn for A. lumbricoides, T. trichiura and hookworms across the 7 trials (countries), age classes, sexes and pre-intervention infection intensities are summarized in Table 4. Overall, the FEC reduction rate for $\operatorname{FECR}(1)$ was the highest for A. lumbricoides $(99.5 \%)$, followed by hookworm $(94.8 \%)$ and $T$. trichiura $(50.8 \%)$. However, there was considerable variation in the FEC reduction rate among the 7 trials, age classes and infection intensities at pre-intervention survey. For $A$. lumbricoides, the FEC reduction rate remained roughly unchanged over these variables, only ranging from 97.8 to $100 \%$. This contrasts with T. trichiura, for which the FEC reduction rate differed between the trials (from 39.2 [Cameroon] to $92.4 \%$ [Ethiopia]), age classes (from 45.4 [B] to $62.7 \%[\mathrm{~A}]$ ) and pre-intervention infection intensity (from 40.0 [high] to $58.7 \%$ [moderate]). There was no difference in the FEC reduction rate between the sexes. For hookworms, only small differences in the FEC reduction rate were observed between the trials, ranging from 87.1 [India] to $100 \%$ [Vietnam]. However, there were only negligible differences between the age classes (from 94.7 [B] to $96.4 \%[\mathrm{C}])$.

Compared to the results of FECR (1), the outputs of FECR (2) resulted in higher values for all three $\mathrm{STH}$, except for $A$. lumbricoides where the FEC reduction rate already showed a ceiling effect $(100 \%)$. Considerable variation in the FEC reduction rate (FECR (2)) occurred with T. trichiura among the trials (from 82.6 [Tanzania] to $99.1 \%$ [Ethiopia]) and pre-intervention infection intensity (from 88.6 [high] to $94.3 \%$ [low]). For hookworms, the differences between the trials were virtually negligible, all indicating a potent effect just short of the maximum 100\% (FECR (2) $>99.3 \%$ ).

The results of FECR (3) mostly yielded comparable or lower values than those from FECR (1). The low values (sometimes negative) can be explained by subjects for whom the post- 


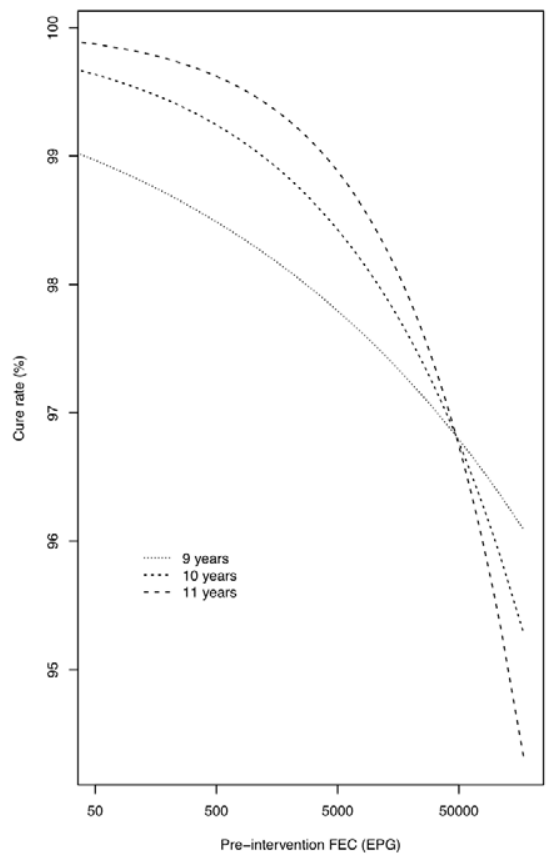

B

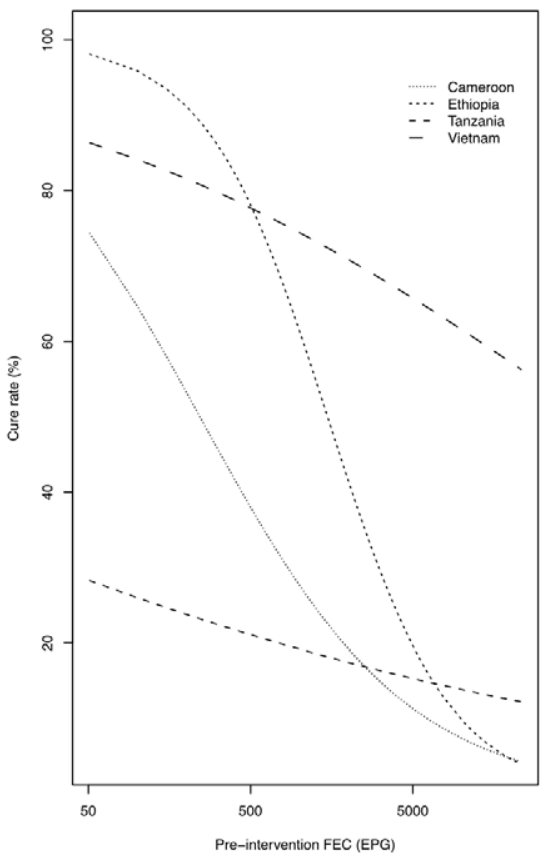

c

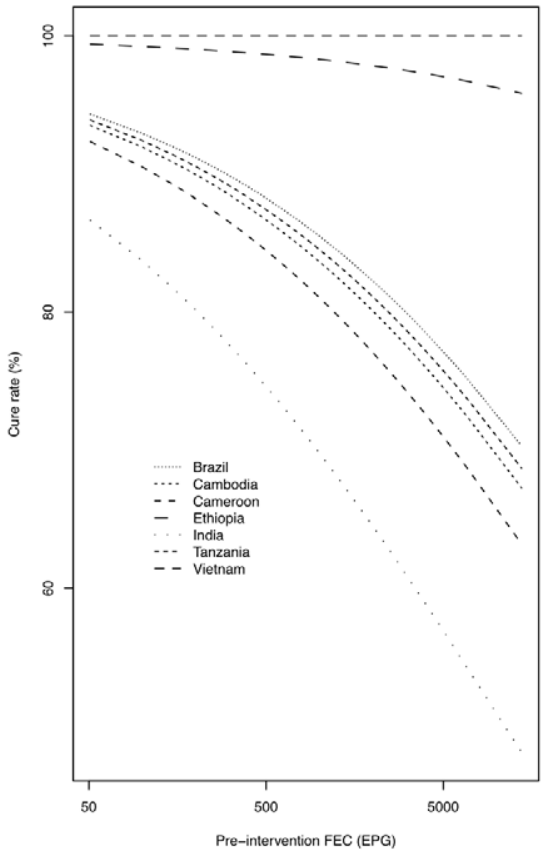

Figure 2. The estimated impact of age, fecal egg count and trial on the cure rate. The estimated impact of age, fecal egg count (FEC) and trial (country) on the cure rate of A. lumbricoides (A), T. trichiura (B) and hookworms (C) derived from a generalized linear models (binomial error). doi:10.1371/journal.pntd.0000948.g002

intervention FEG exceeded the pre-intervention FEC. These subjects contributed to a negative FEC reduction rate which had a significant impact on the final FEC reduction rate calculated with FECR (3). This became apparent in the FEG reduction rate for $A$. lumbricoides, where a Cameroonian male subject of 7 years with a pre-intervention FEC of 100 and a post-intervention FEG of 22,050 EPG, contributed markedly to lowering the overall values for the data-set from the trial in Cameroon (FECR (1): 99.2\%;
FECR (3): 26.0\%). This lowering of FECR (3) compared to FECR (1) for A. lumbricoides also occurred with age class A (FECR (1): $98.9 \%$; FECR (3): $-2.7 \%$ ) and the low pre-intervention infection intensity level (FECR (1): 97.8\%; FECR (3): 66.6\%), but not for the remaining variables. The number of negative individual FEC reduction rates, and the magnitude of the difference between preand post-intervention FEC, both contributed to the discrepancies found for T. trichiura (176 subjects) and hookworms (10 subjects).

Table 3. The arithmetic means of fecal egg counts of soil-transmitted helminths across the seven trials (countries).

\begin{tabular}{|c|c|c|c|c|c|c|}
\hline & \multicolumn{2}{|c|}{ A. Iumbricoides } & \multicolumn{2}{|c|}{ T. trichiura } & \multicolumn{2}{|c|}{ Hookworms } \\
\hline & $\mathbf{N}$ & $\begin{array}{l}\text { Arithmetic mean } \\
\text { (min-max) }\end{array}$ & $\mathbf{n}$ & $\begin{array}{l}\text { Arithmetic mean } \\
\text { (min-max) }\end{array}$ & $\mathbf{n}$ & $\begin{array}{l}\text { Arithmetic mean } \\
\text { (min-max) }\end{array}$ \\
\hline Brazil & 50 & $\begin{array}{l}9230 \\
(100-51250)\end{array}$ & 1 & 50 & 52 & $\begin{array}{l}617 \\
(50-2900)\end{array}$ \\
\hline Cambodia & 5 & $\begin{array}{l}1420 \\
(50-6600)\end{array}$ & 2 & $\begin{array}{l}175 \\
(50-300)\end{array}$ & 127 & $\begin{array}{l}585 \\
(50-4400)\end{array}$ \\
\hline Cameroon & 298 & $\begin{array}{l}12085 \\
(50-170500)\end{array}$ & 386 & $\begin{array}{l}1023 \\
(50-23200)\end{array}$ & 140 & $\begin{array}{l}567 \\
(50-13800)\end{array}$ \\
\hline Ethiopia & 151 & $\begin{array}{l}3443 \\
(100-62500)\end{array}$ & 105 & $\begin{array}{l}420 \\
(100-5200)\end{array}$ & 91 & $\begin{array}{l}326 \\
(100-1650)\end{array}$ \\
\hline India & 21 & $\begin{array}{l}2927 \\
(50-13100)\end{array}$ & 18 & $\begin{array}{l}305 \\
(50-1100)\end{array}$ & 95 & $\begin{array}{l}662 \\
(100-3000)\end{array}$ \\
\hline Tanzania & 279 & $\begin{array}{l}4279 \\
(50-55750)\end{array}$ & 396 & $\begin{array}{l}924 \\
(50-6650)\end{array}$ & 349 & $\begin{array}{l}867 \\
(50-6950)\end{array}$ \\
\hline Vietnam & 148 & $\begin{array}{l}4741 \\
(50-53000\end{array}$ & 138 & $\begin{array}{l}371 \\
(50-4600)\end{array}$ & 58 & $\begin{array}{l}205 \\
(50-2500)\end{array}$ \\
\hline Total & 952 & $\begin{array}{l}6877 \\
(50-170500)\end{array}$ & 1046 & $\begin{array}{l}824 \\
(50-23200)\end{array}$ & 912 & $\begin{array}{l}650 \\
(50-13800)\end{array}$ \\
\hline
\end{tabular}

doi:10.1371/journal.pntd.0000948.t003 


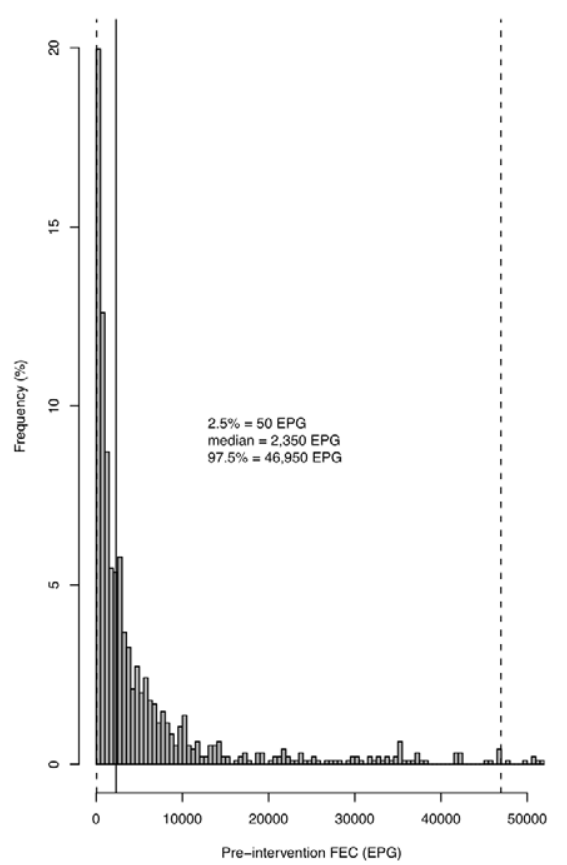

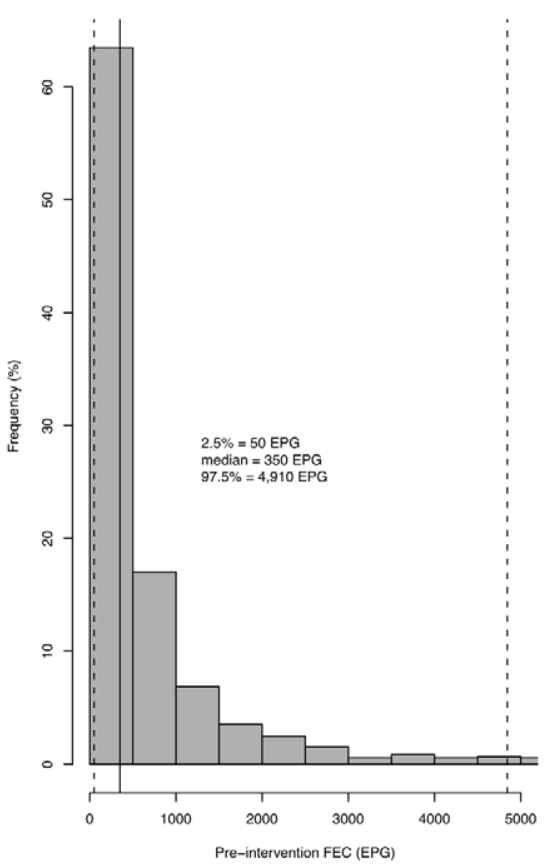

c

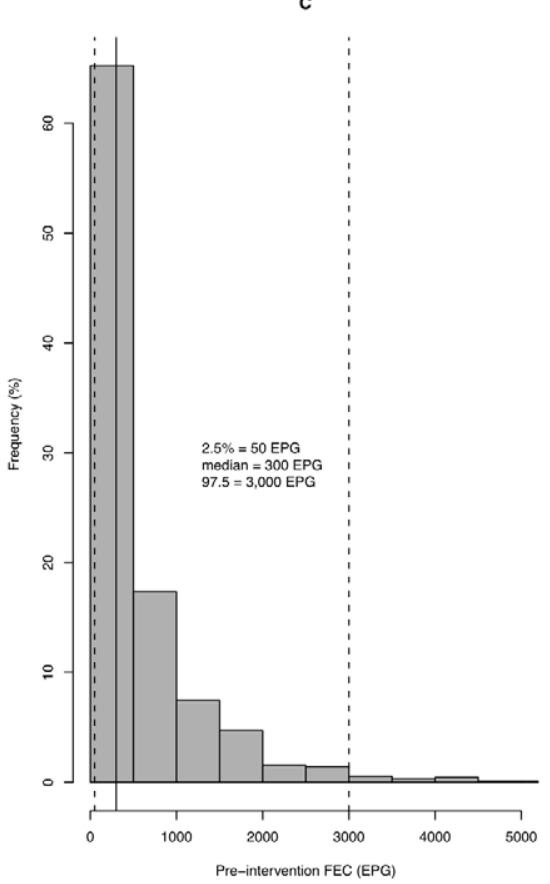

Figure 3. The distribution of the fecal egg count of soil-transmitted helminths. The distribution of the fecal egg count (FEC) of $A$. lumbricoides (A), T. trichiura (B) and hookworms (C). The absolute values of the median, the $2.5^{\text {th }}$ and $97.5^{\text {th }}$ percentile (\%) are provided. In addition, these values are represented as solid (median) and dashed lines (2.5 and 97.5\%).

doi:10.1371/journal.pntd.0000948.g003

\section{Robustness of FECR formulae}

Table 5 summarizes the FEG reduction rates restricted to samples of more than 150 EPG indicating that the results of FECR (1) and FECR (2) remained roughly unchanged. The values from FECR (3) increased and were mostly comparable with those obtained by FECR (1). This change in the results of FECR (3) is due to the exclusion of negative individual FEG reduction rates which mostly occurred among the subjects with low preintervention FEC (see also Table 4). Differences of more than $5 \%$ between the results of FECR (3) and FECR (1) were limited to T. trichiura (country: Cameroon, India, Tanzania and Vietnam; age class: $\mathrm{A}$ and $\mathrm{C}$ ).

\section{Factors associated with FECR}

The assessment of putative factors affecting the results from FECR (3) was restricted to samples containing more than 150 EPG. Due to the limited variation in the FEC reduction rates (FECR (3)) of $A$. lumbricoides across the different variables, this species was not analyzed further. Also, because of the limited number of infected subjects $(<50)$, the trials in Brazil, Cambodia and India were excluded from analyses of $T$. trichiura. For hookworms, and for the same reasons, subjects from the trials in Brazil and Vietnam were not included. Significant differences in the FEC reduction rates between the trials were found for both $T$. trichiura $\left(\chi^{2}{ }_{3}=117.3, p<0.0001\right)$ and hookworms $\left(\chi^{2}{ }_{4}=20.2\right.$, $p=0.0005)$. High pre-intervention FEC of $\mathcal{T}$. trichiura yielded lower FEC reduction rates $(3)\left(\mathrm{R}_{\mathrm{s}}=-0.18, \mathrm{n}=701, \mathrm{p}<0.0001\right)$, but this was not found for hookworm $\left(R_{s}=-0.04, n=601\right.$, $\mathrm{p}=0.34)$. In addition, there was an interaction between the preintervention FEC of $T$. trichiura and trial (country), reflected in the negative correlations in the trials in Cameroon $\left(\mathrm{R}_{\mathrm{s}}=-0.28\right.$, $\mathrm{n}=233, \quad \mathrm{p}<0.0001)$, and Ethiopia, $\left(\mathrm{R}_{\mathrm{s}}=-0.34, \quad \mathrm{n}=72\right.$, $\mathrm{p}=0.0034$ ), but a positive correlation for the trial in Tanzania
$\left(\mathrm{R}_{\mathrm{s}}=+0.28, \mathrm{n}=325, \mathrm{p}<0.0001\right)$ and a non-significant correlation for the trial in Vietnam $\left(\mathrm{R}_{\mathrm{s}}=-0.07, \mathrm{n}=71, \mathrm{p}=0.58\right)$. Host sex and age did not contribute significantly to variation of the results of (FECR (3)) in any of the STH examined.

\section{Discussion}

To our knowledge, the present study is the first to evaluate drug efficacy for STH in school children across different endemic regions using a protocol which was standardized in terms of the treatment (a single-oral $400 \mathrm{mg}$ dose of ALB originating from the same batch), the follow up (between 14 and 30 days after) and the detection technique (the McMaster counting technique). Moreover, efficacy was evaluated by both the CR and the FECR, and compared statistically between the seven trials which took place in geographically disparate parts of the world.

Overall, this study supports previous reports that indicated that single dose ALB treatment is most effective for infection with $A$. lumbricoides, followed by hookworm, but is relatively ineffective for T. trichiura, confirming the efficacy studies reviewed by Bennet and Guyatt [3], and by Keiser and Utzinger [8]. The low efficacy observed for T. trichiura compared to the two other STH, is in keeping with previous studies, where a 3-day dose schedule of ALB has been shown to be necessary to achieve acceptable therapeutic efficacy [3].

At present, the most commonly reported indicator of drug efficacy in this field is the CR [3]. Our results support the view that the GR should not be the recommended parameter, as it is sensitive to variation in the intensity of infection before treatment. The CRs declined in all three STH with increasing intensity of infection (FEC) at the pre-intervention survey. Hence, comparison between populations (countries, villages, schools, etc.) differing in pre-intervention FEC are guaranteed to arrive at different conclusions about drug efficacy. Differences in the outputs of 
Table 4. Fecal egg count reduction across different countries, age classes, sexes and pre-intervention intensities.

\begin{tabular}{|c|c|c|c|c|c|c|c|c|c|c|c|c|}
\hline & \multicolumn{4}{|c|}{ A. lumbricoides } & \multicolumn{4}{|c|}{ T. trichiura } & \multicolumn{4}{|c|}{ Hookworms } \\
\hline & $\mathbf{n}$ & $\begin{array}{l}\text { FECR } \\
(1) \\
(\%)\end{array}$ & $\begin{array}{l}\text { FECR } \\
\text { (2) } \\
\text { (\%) }\end{array}$ & $\begin{array}{l}\text { FECR } \\
\text { (3) } \\
(\%)\end{array}$ & $\mathbf{n}$ & $\begin{array}{l}\text { FECR } \\
(1) \\
(\%)\end{array}$ & $\begin{array}{l}\text { FECR } \\
\text { (2) } \\
\text { (\%) }\end{array}$ & $\begin{array}{l}\text { FECR } \\
\text { (3) } \\
(\%)\end{array}$ & $\mathbf{n}$ & $\begin{array}{l}\text { FECR } \\
(1) \\
(\%)\end{array}$ & $\begin{array}{l}\text { FECR } \\
(2) \\
(\%)\end{array}$ & $\begin{array}{l}\text { FECR } \\
\text { (3) } \\
\text { (\%) }\end{array}$ \\
\hline \multicolumn{13}{|l|}{ Country } \\
\hline Brazil & 50 & 100.0 & 100.0 & 100.0 & $1^{*}$ & 100.0 & 98.0 & 100.0 & 52 & 97.5 & 99.6 & 97.8 \\
\hline Cambodia & $5^{*}$ & 100.0 & 99.6 & 100.0 & $2^{*}$ & 100.0 & 99.2 & 100.0 & 127 & 97.6 & 99.5 & 96.6 \\
\hline Cameroon & 298 & 99.2 & 100.0 & 26.0 & 386 & 39.2 & 93.0 & 34.7 & 140 & 93.0 & 99.2 & 91.9 \\
\hline Ethiopia & 151 & 100.0 & 99.9 & 100.0 & 105 & 92.4 & 99.1 & 93.1 & 91 & 99.7 & 99.6 & 99.9 \\
\hline India & $21^{*}$ & 98.9 & 99.8 & 99.7 & $18^{*}$ & 74.5 & 98.9 & 92.1 & 95 & 87.1 & 99.2 & 81.6 \\
\hline Tanzania & 279 & 100.0 & 99.9 & 99.9 & 396 & 52.0 & 82.6 & -36.2 & 349 & 95.3 & 99.6 & 92.6 \\
\hline Vietnam & 148 & 100.0 & 99.9 & 99.9 & 138 & 92.3 & 98.8 & 86.4 & 58 & 100.0 & 99.3 & 100.0 \\
\hline \multicolumn{13}{|l|}{ Age class } \\
\hline A (4-8) & 215 & 98.9 & 100.0 & -2.7 & 219 & 62.7 & 94.0 & 32.2 & 173 & 94.8 & 99.5 & 95.2 \\
\hline B (9-13) & 669 & 99.8 & 99.9 & 100.0 & 753 & 45.4 & 93.8 & 16.3 & 643 & 94.7 & 99.5 & 92.3 \\
\hline C (14-18) & 68 & 100.0 & 100.0 & 100.0 & 74 & 59.6 & 94.7 & 45.4 & 96 & 96.4 & 99.5 & 97.0 \\
\hline \multicolumn{13}{|l|}{ Sex } \\
\hline Female & 462 & 100.0 & 99.9 & 99.9 & 503 & 50.3 & 94.6 & 9.3 & 393 & 95.2 & 99.5 & 94.2 \\
\hline Male & 490 & 99.0 & 99.9 & 54.9 & 543 & 51.2 & 93.2 & 33.2 & 519 & 94.5 & 99.5 & 92.8 \\
\hline \multicolumn{13}{|c|}{ Pre-intervention infection intensity } \\
\hline Low & 662 & 97.8 & 99.9 & 66.6 & 823 & 40.2 & 94.3 & 11.9 & 859 & 93.9 & 99.4 & 93.2 \\
\hline moderate & 270 & 100.0 & 100.0 & 100.0 & 215 & 58.7 & 92.2 & 58.7 & 40 & 97.6 & 99.9 & 97.1 \\
\hline High & 20 & 99.5 & 100.0 & 99.6 & 8 & 40.0 & 88.6 & 40.1 & 13 & 95.9 & 99.9 & 96.4 \\
\hline Total & 952 & 99.5 & 99.9 & 76.8 & 1046 & 50.8 & 93.9 & 21.7 & 912 & 94.8 & 99.5 & 93.4 \\
\hline
\end{tabular}

FECR(1): group based and arithmetic mean; FECR(2): group based and geometric mean; FECR(3): individual based and arithmetic.

*Due to the low number of infected subjects $(<50)$, the trials conducted in these countries were excluded from further analysis.

doi:10.1371/journal.pntd.0000948.t004

calculations based on processing quantitative data in different ways also showed variation that requires careful review if standard operating procedures for data processing are to be adopted. The observation that therapeutic efficacies based on arithmetic means were mostly lower than those based on geometric means is in agreement with other studies [15], and arises because the arithmetic means captures the variation more effectively, while the geometric means compress the data such that efficacies are highly overestimated. Our exploratory analysis of different statistical approaches for analyzing data also indicates that FECR based on individuals was highly affected by excluding subjects with pre-intervention FEC below 150 EPG. Therefore, we conclude that the group based formula using an arithmetic mean is the best summary statistic to employ in analysis of therapeutic efficacy in future large scale drug administration trials, since it represents a robust indicator that is sensitive to changes in drug efficacy.

The efficacy (CR and FEC reduction rate) varied widely across the trials, except for $A$. lumbricoides. Possible explanations for the observed differences include (1) treatment history, (2) geographic differences within STH species, (3) fecal consistency and (4) diet. It is therefore pertinent to comment on each. Although the lowest efficacies for T. trichiura (Cameroon and Tanzania) and hookworms (India) were obtained in countries with a treatment history, the observed low efficacies are not likely to be attributable to large scale anthelmintic treatment in Cameroon and India. In these countries, a comparison between different study sites with a history of large scale anthelmintic treatment (Cameroon: Loum; India: Vellore) and without such a history (Cameroon: Yoyo; India:
Thiruvanamalai) indicated that these large scale programs did not result in a reduced efficacy compared to sites were they were absent (data not shown and to be published separately). For Tanzania, the impact of large scale anthelmintic treatment programs could be ruled out, as studies before and during these interventions have shown similar drug efficacy figures for $T$. trichuria $[16,17]$.

Current molecular studies indicate that geographical differences exist within STH species [18,19]. For T. trichiura varying anthelmintic efficacy has been suggested to be attributable to the presence/absence of the $\beta$-tubulin codon 200 polymorphism that has been linked to BZ resistance [20]. Strain differences have been demonstrated in some species with different drug tolerance as assessed both by efficacy and molecular studies [20,21]. Nevertheless, the exact impact of genetic differences within the $3 \mathrm{STH}$ in this study on the efficacy of specific anthelmintics remains speculative. Of note, even at a higher taxonomic level, information on the relative therapeutic efficacy of a single dose ALB on $\mathcal{N}$. americanus and $A$. duodenale is scarce, this despite the distinct and well known biological differences between these hookworms [2224]. FEC was calculated in the current study without compensation for fecal consistency. It is well recognized that well-formed stools can concentrate helminth eggs, compared to looser or diarrheic feces where they are diluted [25], thus confounding assessment of drug efficacy. Finally, the diet of subjects varied considerably across the seven participating countries. Differences in the quality of food consumed would have created differences in fat and roughage content and/or increased the rate of passage of 
Table 5. Fecal egg count reduction for samples with a pre-intervention FEC of more than 150 EPG.

\begin{tabular}{|c|c|c|c|c|c|c|c|c|c|c|c|c|}
\hline & \multicolumn{4}{|c|}{ A. lumbricoides } & \multicolumn{4}{|c|}{ T. trichiura } & \multicolumn{4}{|c|}{ Hookworms } \\
\hline & $\mathbf{n}$ & $\begin{array}{l}\text { FECR } \\
(1) \\
(\%)\end{array}$ & $\begin{array}{l}\text { FECR } \\
\text { (2) } \\
(\%)\end{array}$ & $\begin{array}{l}\text { FECR } \\
\text { (3) } \\
\text { (\%) }\end{array}$ & $\mathbf{n}$ & $\begin{array}{l}\text { FECR } \\
(1) \\
(\%)\end{array}$ & $\begin{array}{l}\text { FECR } \\
\text { (2) } \\
(\%)\end{array}$ & $\begin{array}{l}\text { FECR } \\
\text { (3) } \\
\text { (\%) }\end{array}$ & $\mathbf{n}$ & $\begin{array}{l}\text { FECR } \\
(1) \\
(\%)\end{array}$ & $\begin{array}{l}\text { FECR } \\
\text { (2) } \\
(\%)\end{array}$ & $\begin{array}{l}\text { FECR } \\
\text { (3) } \\
\text { (\%) }\end{array}$ \\
\hline \multicolumn{13}{|l|}{ Country } \\
\hline Brazil & $47^{*}$ & 100.0 & 100.0 & 100.0 & $0^{*}$ & - & - & - & $46^{*}$ & 97.5 & 99.6 & 97.5 \\
\hline Cambodia & $1^{*}$ & 100.0 & 100.0 & 100.0 & $1^{*}$ & 100.0 & 99.7 & 100.0 & 100 & 97.7 & 99.6 & 96.7 \\
\hline Cameroon & 266 & 99.8 & 100.0 & 100.0 & 233 & 39.9 & 93.4 & 50.4 & 71 & 93.6 & 99.5 & 95.1 \\
\hline Ethiopia & 145 & 100.0 & 99.9 & 100.0 & 72 & 92.3 & 99.2 & 92.6 & 66 & 99.6 & 99.7 & 99.8 \\
\hline India & $17^{*}$ & 98.9 & 99.9 & 99.6 & $11^{*}$ & 72.0 & 99.1 & 87.0 & 83 & 87.8 & 99.3 & 84.2 \\
\hline Tanzania & 266 & 100.0 & 100.0 & 99.9 & 325 & 58.3 & 86.6 & 36.4 & 281 & 95.4 & 99.7 & 93.1 \\
\hline Vietnam & 130 & 100.0 & 99.9 & 99.9 & 71 & 93.1 & 99.2 & 88.0 & $19^{*}$ & 100.0 & 99.7 & 100.0 \\
\hline \multicolumn{13}{|l|}{ Age class } \\
\hline A (4-8) & 196 & 99.9 & 100.0 & 99.8 & 153 & 65.1 & 94.8 & 57.2 & 130 & 94.7 & 99.6 & 94.4 \\
\hline B (9-12) & 613 & 99.8 & 100.0 & 99.9 & 515 & 48.4 & 94.1 & 51.8 & 460 & 94.9 & 99.6 & 93.2 \\
\hline C (13-18) & 63 & 100.0 & 100.0 & 100.0 & 45 & 60.2 & 94.4 & 46.4 & 76 & 96.4 & 99.6 & 97.1 \\
\hline \multicolumn{13}{|l|}{ Sex } \\
\hline Female & 428 & 100.0 & 100.0 & 99.9 & 343 & 54.0 & 94.7 & 57.7 & 286 & 95.2 & 99.6 & 93.8 \\
\hline Male & 444 & 99.7 & 100.0 & 99.9 & 370 & 53.0 & 93.8 & 48.0 & 380 & 94.8 & 99.6 & 94.0 \\
\hline \multicolumn{13}{|c|}{ Pre-intervention infection intensity } \\
\hline Low & 582 & 99.9 & 99.9 & 99.9 & 490 & 49.0 & 95.1 & 50.2 & 613 & 94.1 & 99.6 & 93.6 \\
\hline Moderate & 270 & 100.0 & 100.0 & 100.0 & 215 & 58.7 & 92.2 & 58.7 & 40 & 97.6 & 99.9 & 97.1 \\
\hline High & 20 & 99.5 & 100.0 & 99.6 & 8 & 40.0 & 88.6 & 40.1 & 13 & 95.9 & 99.9 & 96.4 \\
\hline Total & 872 & 99.9 & 100.0 & 99.9 & 713 & 53.5 & 94.3 & 52.7 & 666 & 95.0 & 99.6 & 93.9 \\
\hline
\end{tabular}

FECR(1): group based and arithmetic mean; FECR(2): group based and geometric mean; FECR(3): individual based and arithmetic. *Due to the low number of infected subjects $(<50)$, the trials conducted in these countries were excluded from further analysis.

doi:10.1371/journal.pntd.0000948.t005

substances through the gastrointestinal tract. This may have reduced the period over which ALB could have acted on the parasites, thereby reducing efficacy [26-28].

Kopp et al. [29] demonstrated that a reduction in adult canine hookworm (A. caninum) counts following chemotherapy did not always yield a reduction in FEC, due to an increase in fecundity among the small residual worm population that survived the anthelmintic treatment (i/e., density dependent fecundity), consequently confounding the FECR. As described by Kotze and Kopp [30], density dependent effects could be manifested in a FECR as a reduced drug efficacy for subjects with higher pre-intervention FEC. However, this did not occur in the present study for $A$. lumbricoides and hookworm. For T. trichiura, the efficacy did decrease with increasing pre-intervention FEC, but this should be interpreted with some caution. This effect was not consistent across the different trials (e.g., no correlation in Vietnam but a positive correlation in Tanzania), suggesting that other factors as discussed above may have confounded this result. It is also possible that increases in FEG may have arisen because of the inability of ALB to cure infections during the pre-patent period (with an onset of patency after the pre-intervention egg count time point). This is a complication that cannot be avoided in studies taking place in endemic areas where transmission occurs daily because of soil and food contaminated with infective stages of the parasites, and is not interrupted in the population during the period of study. Finally, a negative correlation between the FEC and efficacy is expected, as the probability of having a FEC of zero after treatment in the follow-up survey, consequently a FECR of $100 \%$, will be higher for low FEC than for high FEC before the administration of the drug.

Our findings emphasize a need to adhere to strict standard operating procedures and methodologies, and to change the WHO recommended threshold levels for the efficacy of ALB [31], where a FEG reduction rate below $70 \%$ in the case of $A$. lumbricoides or below $50 \%$ for the hookworms are the currently accepted thresholds. We recommend that in future monitoring of single-dose ALB-dependent control programs a minimum FEC reduction rate (based on arithmetic means) of $>95 \%$ for $A$. lumbricoides and $>90 \%$ for hookworms are appropriate thresholds, and that efficacy levels below this should raise concern. The great variability of the FECR for T. trichiura and the relatively low efficacy of ALB, confirmed in this present study, indicate that it is not possible to propose an efficacy threshold for this parasite based on our data.

In conclusion, the present study is the first to evaluate drug efficacy of a single-oral dose of ALB on such a scale and across three continents. The results confirm the therapeutic efficacy of this treatment against $A$. lumbricoides and hookworms, and the low efficacy against $T$. trichiura. Efficacy varied widely across the seven different trials, particularly in the case of T. trichiura and it remains unclear which factors were principally responsible for this variation, although pre-intervention FEC and age played clear roles in this respect. The FEC reduction rate based on arithmetic means is the best available indicator of drug efficacy, and should be adopted in future monitoring and evaluation studies of large scale anthelmintic treatment programs. Finally, our findings 
emphasize the need to revise the WHO recommended efficacy threshold for single dose ALB treatments.

\section{Supporting Information}

Ghecklist S1 CONSORT Checklist

Found at: doi:10.1371/journal.pntd.0000948.s001 (0.22 MB DOC)

Protocol S1 Trial Protocol

Found at: doi:10.1371/journal.pntd.0000948.s002 (1.17 MB PDF)

\section{Acknowledgments}

The following institutions and persons are thanked for their assistance and help:

Brazil - We would like to acknowledge the contributions of Renata Caldeira Diniz, Simone Aparecida Pinto, Michele Silva de Matos and Adriana Pereira da Silva in collection and analysis of samples.

Cambodia - Dr Muth Sinuon (National Center for Parasitology, Entomology and Malaria, GNM), Dr Philippe Guyant (Partners for Development, PfD), Mrs Hem Sopheak (Institut Pasteur in Cambodia) and the various members of staff of the Medical Microbiology Laboratory (Institut Pasteur in Cambodia), National Center for Parasitology, Entomology and Malaria and Partners for Development who helped to facilitate the study.

\section{References}

1. World Health Organization (2005) Deworming for health and development. Report of the third global meeting of the partners for parasitic control. WHO/ CDS/CPE/PVC/2005.14, World Health Organization, Geneva.

2. Albonico M, Allen H, Chitsulo L, Engels D, Gabrielli AF, et al. (2008) Controlling soil-transmitted helminthiasis in pre-school-age children through preventive chemotherapy. PLoS Negl Trop Dis 2: e126.

3. Bennett A, Guyatt H (2000) Reducing intestinal nematode infection: efficacy of albendazole and mebendazole. Parasitol Today 16: 71-74.

4. Wolstenholme AJ, Fairweather I, Prichard R, von Samson-Himmelstjerna G, Sangster NC (2004) Drug resistance in veterinary helminths. Trends Parasitol 20: $469-476$.

5. Albonico M, Engels D, Savioli L (2004) Monitoring drug efficacy and early detection of drug resistance in human soil-transmitted nematodes: a pressing public health agenda for helminth control. Int J Parasitol 34: 1205-1210.

6. Geerts S, Gryseels B (2001) Anthelmintic resistance in human helminths: a review. Trop Med Int Health 6: 915-921.

7. De Clercq D, Sacko M, Behnke JM, Gilbert F, Dorny P, et al. (1997) Failure of mebendazole in treatment of human hookworm infections in the Southern Region of Mali. Am J Trop Med Hyg 57: 25-30.

8. Keiser J, Utzinger J (2008) Efficacy of current drugs against soil-transmitted helminth infections - systematic review and meta-analysis. JAMA 299: 1937-1948.

9. Levecke B, De Wilde N, Vandenhoute E, Vercruysse J (2009) Field validity and feasibility of four techniques for the detection of Trichuris in simians: a model for monitoring drug efficacy in public health? PLoS Negl Trop Dis 3(1): e366.

10. Coles GC, Bauer C, Borgsteede FH, Geerts S, Klei TR, et al. (1992) World Association for the Advancement of Veterinary Parasitology (W.A.A.V.P.) methods for the detection of anthelmintic resistance in nematodes of veterinary importance. Vet Parasitol 44: 35-44.

11. Coles GC, Jackson F, Pomroy WE, Samson-Himmelstjerna G, Silvestre A, et al. (2006) The detection of anthelmintic resistance in nematodes of veterinary importance. Vet Parasitol 136: 167-185.

12. Ministry of Agriculture, Fisheries and Food (1986) Manual of veterinary parasitological laboratory techniques (Reference Book; 418), 3rd ed. London: Her Majesty's Stationery Office (HMSO). 160 p.

13. Montresor A, Crompton DWT, Hall H, Bundy DAP, Savioli L (1998) Guidelines for the evaluation of soil-transmitted helminthiasis and schistosomiasis at community level. A guide for control programme managers. WHO/ CTD/SIP98.1, World Health Organization, Geneva.

14. Crawley MT (1993) GLIM for Ecologists. Oxford: Blackwell Scientific Press. ix $+380 \mathrm{p}$.

15. Dobson RJ, Sangster NC, Besier RB, Woodgate RG (2009) Geometric means provide a biased efficacy result when conducting a fecal egg count reduction test (FECRT). Vet Parasitol 161: 162-167.

16. Albonico M, Smith PG, Hall A, Chwaya HM, Alawi KS (1994) A randomised controlled trial comparing mebendazole $500 \mathrm{mg}$ and albendazole $400 \mathrm{mg}$
Cameroon - Luogbou Nzu Deguy D'or, Wapi Christelle and Ndogmo Calvine for their assistance with sampling.

Ethiopia - The elementary school teachers and students (study subjects) of Jiren no 1 with special thanks to Mr. Gulte Z.Agegnehu, Director of the school, Mr. Mio Ayana and other senior technicians working in Dept. of Medical Laboratory Science, Jimma University, for the field sample collection and laboratory examination.

India - Dr. Rajiv Sarkar, Dr. Shantidani Minz and Dr. Jayaprakash Muliyil for helping with organizing the sample collection and Ms. Sheela Roy, Dr. Sitara Rao Ajjampur and the field and laboratory teams for collecting and analyzing the samples.

Vietnam - Dr Tran Cong Dai (WHO Country Office) that greatly facilitated the communications with Nimpe and provided technical support.

Zanzibar - The laboratory technicians of the Parasitology Laboratory, Public Health Laboratory, Ivo de Carneri for their role in the collection and analysis of samples.

\section{Author Contributions}

Conceived and designed the experiments: JV JMB MA ACK JSM AM BL. Performed the experiments: SMA CA JMB DE BG NTVH GK DK ZM MVP LS L-ATT DTCT AZ. Analyzed the data: JV JMB BL. Wrote the paper: JV JMB BL. Revision of the paper: MA JMB ACK JSM AM. Recruitment and enrolment of patients and parasitological examinations: SMA CA JMB DE BG NTVH GK DK ZM MVP LS L-ATT DTCT AZ.

against Ascaris, Trichuris and the hookworms. Trans R Soc Trop Med Hyg 88: 585-589.

17. Albonico M, Bickle Q, Ramsan M, Montresor A, Savioli L, et al. (2003) Efficacy of mebendazole and levamisole alone or in combination against intestinal nematode infections after repeated targeted mebendazole treatment in Zanzibar. Bull WHO 81: 343-352.

18. Hu M, Chilton NB, El-Osta YGA, Gasser RB (2003) Comparative analysis of mitochondrial genome data for Necator americanus from two endemic regions reveals substantial genetic variation. Int J Parasitol 33: 955-963.

19. Leles D, Araújo A, Vicente AC, Iñiguez AM (2009) Molecular diagnosis of ascariasis from human feces and description of a new Ascaris sp. genotype in Brazil. Vet Parasitol 163: 167-170.

20. Diawara A, Drake LS, Suswillo RR, Kihara J, Bundy DA, et al. (2009) Assays to detect B tubulin Codon 200 polymorphism in Trichuris trichiura and Ascaris lumbricoides. PLoS Negl Trop Dis 3: e397.

21. Marti H, Haji HJ, Savioli L, Chwaya HM, Mgeni AF, et al. (1996) A comparative trial of a single-dose ivermectin versus three days of albendazole for treatment of Strongyloides stercoralis and other soil-transmitted helminth infections in children. Am J Trop Med Hyg 55: 477-481.

22. Hoagland KE, Schad GA (1978) Necator americanus and Ancylostoma duodenale: life history parameters and epidemiological implications of two sympatric hookworms of human. Exp Parasitol 44: 36-49.

23. El-Masry NA, Trabolsi B, Bassily S, Farid Z (1983) Albendazole in the treatment of Ancylostoma duodenale and Ascaris lumbricoides infections. Trans R Soc Trop Med Hyg 77: 160-161.

24. Sacko M, De Clercq D, Behnke JM, Gilbert FS, Dorny P, et al. (1999) Comparison of the efficacy of mebendazole, albendazole and pyrantel in treatment of human hookworm infections in the southern region of Mali, West Africa. Trans R Soc Trop Med Hyg 93: 195-203.

25. World Health Organization (1961) CCTA/WHO African conference on ancylostomiasis, Brazzaville 22-29 August 1961. Technical Report Series No. 225 .

26. McKellar QA, Scott EW (1990) The benzimidazole anthelmintic agents. A review. J Vet Pharmacol Ther 13: 223-247.

27. Dayan AD (2003) Albendazole, mebendazole and praziquantel. Review of nonclinical toxicity and pharmacokinetics. Acta Trop 86: 141-159.

28. Sanchez SF, Alvarez LI, Lanusse CE (1996) Nutritional condition affects the disposition kinetics of albendazole in cattle. Xenobiotica 25: 307-320.

29. Kopp SR, Coleman GT, McCarthy JS, Kotze AC (2008) Application of in vitro anthelmintic sensitivity assays to canine parasitology: detecting resistance to pyrantel in Ancylostoma caninum. Vet Parasitol 152: 284-293.

30. Kotze AC, Kopp SR (2009) The potential impact of density dependent fecundity on the use of the fecal egg count reduction test for detecting drug resistance in human hookworms. PLoS Negl Trop Dis 2: e297.

31. World Health Organization (1999) Report of the WHO informal consultation on monitoring of drug efficacy in the control of schistosomiasis and intestinal nematodes. WHO/CDS/CPC/SIP/99.1, World Health Organization, Geneva. 